\title{
Multimodal treatment for acute empyema based on the patient's condition, including patients who are bedridden: A single center retrospective study
}

Tomoki Nishida, $M D^{1}$, Yuto Igarashi, $M D^{2}$, Yuma Suno, $M D^{2}$, Takaaki Murata, $\mathrm{MD}^{2}$, Katsunori Miyake, $M D^{2}, \mathrm{PhD}^{2}$, Naoko Isogai, $M^{2}$, Rai Shimoyama, $M D^{2}$, Jun Kawachi, MD $^{2}$, Hiroyuki Kashiwagi, $M D^{2}$, Toshitaka Tsukiyama, $M D^{3}$, Ryuta Fukai, MD, PhD ${ }^{1}$

T Nishida, Y lgarashi, Y Suno, T Murata, K Miyake, N Isogai, R Shimoyama, J Kawachi, H Kashiwagi, T Tsukiyama, R Fukai. Multimodal treatment for acute empyema based on the patient's condition, including patients who are bedridden: A single center retrospective study. Can J Respir Ther 2021;57:143-146. doi: 10.29390/cjrt-2021-032.

\begin{abstract}
Background: The incidence of acute empyema has increased in various countries; some elderly patients with acute empyema have contraindications for surgery under general anesthesia. Therefore, suitable management based on a patient's clinical condition is required.

Methods: We evaluated the different surgical and nonsurgical therapeutic approaches available for patients with acute empyema. This was a retrospective study of 57 patients with acute empyema who received treatment in our department between May 2015 and February 2019. For patients who did not initially improve with drainage or drainage combined with fibrinolytic therapy, surgery, or additional percutaneous drainage was performed based on their general condition. We compared several clinical factors pertaining to the patients who underwent surgical versus nonsurgical treatment.

Results: Our study showed that the patients with a performance status of 0-2 and an American Society of Anesthesiologists physical status classification of class II or lower underwent surgery safely without major operative complications. The combination of repeated drainage of the pleural cavity and fibrinolytic therapy appeared to be a reasonable nonsurgical management option for patients in poor overall condition.

Conclusion: For an aging population, we think that the combination of repeated pleural cavity drainage procedures and fibrinolytic therapy is a reasonable nonsurgical strategy for the management of patients with acute empyema.
\end{abstract}

Key Words: acute empyema; CT-guided drainage; fibrinolytic therapy; elderly patient

\section{INTRODUCTION}

The prevalence of acute empyema among elderly patients has increased in several countries [1, 2], including Japan, and has led to a necessary increase in appropriate treatment options. The management of acute empyema in elderly patients has been accompanied by several problems. For example, the overall condition of elderly patients is often poor and, for most patients, surgery under general anesthesia is not recommended. Determining the time of the onset of illness is also often difficult because of problems obtaining an accurate medical history in some elderly patients who might not remember their symptoms or who have dementia. Therefore, arriving at an accurate determination of the stage of empyema is difficult, although stage-dependent therapy for acute empyema has gradually become the standard of care [3-5].

Because of the problems we have described, we first attempted to insert a drainage tube into the pleural cavity, regardless of the stage of empyema, and then considered the necessity of additional treatment, considering the clinical course of the patient. We discussed each of those patients who we thought needed additional treatment in our daily conference to decide if we should provide surgical or nonsurgical treatment. Computed tomography (CT) guided drainage has been selected more often as additional treatment for elderly patients than surgery because of the poor general condition of these patients. Sokouti et al. [6] found success rates of surgical and nonsurgical treatment of $98.2 \%$ and $27.1 \%$, respectively. The mortality rate of surgical cases after the failure of a single percutaneous drainage procedure after insertion of a chest tube was $1.8 \%$ [6]. To our best knowledge, no reports have been published on repeated percutaneous drainage for acute empyema.

In this study, we retrospectively evaluated the therapeutic approaches used for patients with acute empyema at our hospital and examined the trends and outcomes of both surgical and nonsurgical treatments.

\section{METHODS}

\section{Study participants}

This was a retrospective study of 57 patients with acute empyema who had undergone treatment in our department between May 2015 and February 2019. We collected their data from March 1, 2019 to April 30, 2019. The study included patients who were hospitalized for acute empyema or parapneumonic pleural effusion, while patients with postoperative pulmonary fistula or empyema associated with bronchial fistula were excluded.

Our institution does not turn away any patient, and the annual number of patients delivered by ambulance is greater than 10,000 . Accordingly, we often provide medical care to patients who are bedridden and found difficult to treat at other institutions. In this study, 46 of $57(80.7 \%)$ patients were admitted from the emergency room. Since our

\footnotetext{
${ }^{1}$ Department of General Thoracic Surgery, Shonan Kamakura General Hospital, Kamakura, Japan

${ }^{2}$ Department of General Surgery, Shonan Kamakura General Hospital, Kamakura, Japan

${ }^{3}$ Department of Interventional Radiology, Shonan Kamakura General Hospital, Kamakura, Japan

Correspondence: Tomoki Nishida, 1370-1 Okamoto, Kamakura, Kanagawa, Japan. Tel:+81-467-46-1717, E-mail: tmk-n.322@nifty.com
}

Published online at https://www.cjrt.ca on 20 October 2021 
hospital is a large-volume regional emergency hospital, the proportion of elderly patients is higher than the proportion seen in other institutions, and we have treated more elderly patients in poor general condition with acute empyema than other institutions.

Because this study did not include any invasive intervention for any of the study patients, we did not obtain informed consent from all the patients. However, based on the ethical guidelines of Japan, we provided a means to opt out of the study on our website. The study was approved by the Shonan Kamakura General Hospital Institutional Review Board (TGE01416-024).

\section{Treatment}

Antibiotics were administered to all the study patients, and the first drainage method used for pleural effusion was percutaneous chest tube insertion $(n=44)$ or image-guided (CT or ultrasound) pigtail catheter insertion $(n=13)$. The chest tube consisted of a double-lumen 20- or 24-Fr catheter and was usually inserted by a surgeon. If the CT findings indicated that chest tube insertion would be difficult, as seen in patients with a pleural effusion located in the dorsal or interlobar spaces or adjacent to the mediastinum, an 8-to 14-Fr pigtail catheter was selected for the procedure. Image-guided drainage was always performed by an interventional radiologist (Figure 1); however, a few severely ill patients who required emergency drainage underwent ultrasound-guided insertion of the catheter performed by a thoracic surgeon. Of the 13 patients who received a pig-tail catheter, 11 underwent CT-guided and two underwent ultrasound-guided insertion.

Residual pleural effusion was examined by a second CT examination 2 or 3 days after the first drainage procedure. Forty of $57(70.2 \%)$ patients received urokinase fibrinolytic therapy $(60,000$ units per day) for 3 days after insertion of the first drainage tube and were examined by a second CT examination after fibrinolytic therapy. Some patients who had not improved following the first drainage procedure were considered for surgery after considering age, past medical history, Eastern Cooperative Oncology Group (ECOG) performance status (PS), and other factors. Other patients who had not improved after the first drainage procedure underwent additional CT-guided drainage less than 2 days after the second CT examination. This procedure was performed by an interventional radiologist, who used an 8-to 14-Fr pigtail catheter that was inserted with the use of the same method employed for the first drainage procedure and was combined with fibrinolytic therapy. Furthermore, if empyema was found to be associated with a pulmonary-pleural fistula during the clinical course, bronchial occlusion was performed with the use of an Endobronchial Watanabe Spigot (EWS).

Antibiotic treatment was stopped in the patients when their white blood cell counts normalized, and their C-reactive protein levels decreased to less than $5.0 \mathrm{mg} / \mathrm{dL}$.

\section{FIGURE 1}

Representative image of CT-guided drainage. Insertion of a pig-tail catheter for pleural effusion in the dorsal space (a), and adjacent to the mediastinum (b).

(a)



(b)

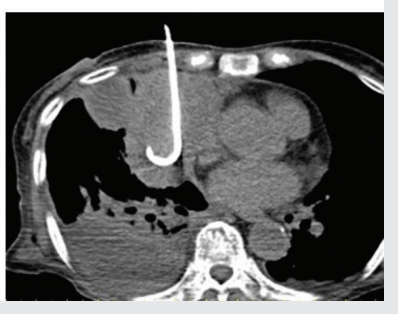

\section{Assessments}

Data on the patients' characteristics, any other relevant factors, and treatment methods were collected retrospectively from the clinical records. In patients who did not improve after the first drainage procedure, we compared the age, sex, recovery period, hospitalization period, ECOG PS, and the American Society of Anesthesiologists physical status classification (ASA-PSC) of the patients who received surgical treatment versus those who received nonsurgical treatment. The recovery period was defined as starting the day when a patient was admitted and ending the day when antibiotic treatment was stopped. The ECOG PS as described 40 years ago was used as follows: grade $0=$ completely active, $1=$ unable to perform physically strenuous activity, 2 = unable to carry out any work activities, $3=$ only capable of limited self-care, $4=$ completely confined to bed, and $5=$ dead [7]. The ASA-PSC published in 1963 was used as follows: Class I = normal healthy patient, II = patient with mild systemic disease, III = patient with severe systemic disease, IV = patient with severe life-threatening systemic disease, and $\mathrm{V}=$ moribund patient not expected to survive without surgery [8]. Bedridden elderly patients without severe disease were defined as unclassifiable for the ASA-PSC $(n=6)$.

\section{Statistical analysis}

Continuous variables (age, PS, recovery period, and hospitalization period) between the 2 treatment groups were compared by the MannWhitney $U$ test, and categorical variables (sex and ASA-PSC) were compared by the $\chi^{2}$ test. Statistical analysis was performed by the EZR software program (Easy R ver.3.6.1, Saitama Medical Center, Jichi Medical University, Saitama, Japan) [9].

\section{RESULTS}

The characteristics of the patients are shown in Table 1 . The median age of the patients was 79.0 (range 45-95) years and included 44 men (77.2\%) and 13 women (22.8\%). Two of 57 patients (3.5\%) died of sepsis within 2 days after the first drainage tube was inserted, whereas all other patients improved (cure rate 96.5\%). However, 7 patients died during hospitalization after resolution of their empyema, with a resulting hospital mortality rate of $15.8 \%$. These patients died because of the following: aspiration pneumonia $(n=1)$, urinary tract infection $(n=1)$, cerebral infarction $(n=1)$, and advanced age $(n=4)$; the patients who died of advanced age had been waiting for transfer to a nursing home and were not resuscitated before death. There were 25 patients $(43.9 \%)$ with dysphagia, and their source of the infection leading to empyema was aspiration pneumonia, which is a common problem in a geriatric population.

The details of the patients $(n=57)$ are summarized in Figure 2 , as follows: $27(47.4 \%)$ patients improved with a single drainage procedure, with a median recovery and hospitalization period of 12.0 (range 6-37) days and 26.0 (range 10-66) days, respectively; among the remaining 28 $(49.1 \%)$ patients, $12(21.1 \%)$ underwent open surgery $(n=2)$ or video-assisted thoracic surgery (VATS) $(n=10)$, and $16(28.1 \%)$ received additional CT-guided insertion of a drainage tube, including 3 (5.3\%) who underwent bronchial occlusion with an EWS.

We show the characteristics of patients, who were classified according to treatment, as surgical versus nonsurgical in Table 2 . The median

\section{TABLE 1}

\section{Characteristics of the patients in the study $(n=57)$}

\begin{tabular}{lcc}
\hline Characteristic & $\boldsymbol{n}$ & $\%$ \\
\hline Sex & & \\
Male & 44 & 77.2 \\
Female & 13 & 22.8 \\
Side & & \\
Right & 34 & 59.6 \\
Left & 23 & 40.4 \\
Outcome & & \\
Cure & 55 & 96.5 \\
Death & 2 & 3.5 \\
\hline
\end{tabular}

Note: Age, years (range) 79.0 (45-95) 


\section{FIGURE 2}

Flowchart of the course of patients from acute empyema after the first percutaneous procedure showing the recovery and hospitalization periods of patients undergoing and those not undergoing surgical treatment.

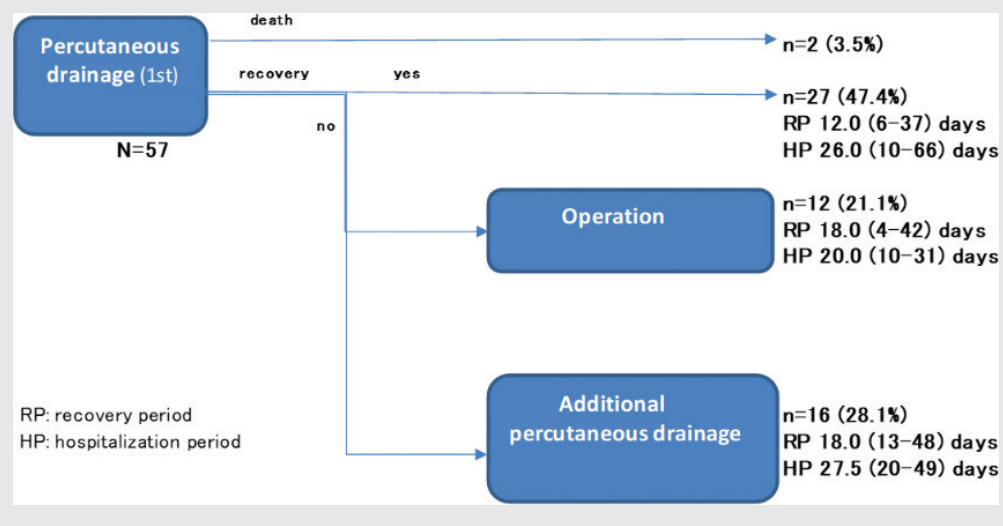

\section{TABLE 2}

\section{Comparisons between patients undergoing and those not undergoing surgical treatment}

\begin{tabular}{|c|c|c|c|}
\hline Variables & Surgical treatment $(n=12)$ & Nonsurgical treatment $(n=16)$ & $P$ \\
\hline Age, y & $65.0(45-87)$ & $81.5(47-95)$ & 0.027 \\
\hline Male & $10.0(83.3)$ & $10.0(62.5)$ & 023 \\
\hline Performance status & $1.0(0-2)$ & $2.5(0-4)$ & 0.0025 \\
\hline \multicolumn{4}{|c|}{ American Society of Anesthesiologists physical status classification } \\
\hline 1 & $1.0(8.3)$ & $1(6.3)$ & - \\
\hline II & $11(91.7)$ & $5(31.3)$ & - \\
\hline III & 0 & $4(25.0)$ & - \\
\hline IV-VI & 0 & 0 & - \\
\hline Recovery period, days & $18.0(4-42)$ & $18.0(113-48)$ & 0.53 \\
\hline Hospitalization period, days & $20.0(10-31)$ & $27.5(20-49)$ & 0.09 \\
\hline
\end{tabular}

Values are expressed as medians (range) or $\mathrm{n}(\%)$.

ages were 65.0 versus 81.5 years $(p=0.027)$, respectively, and median PS was $1.0(0-2)$ versus $.2 .5(0-4)(p=0.025)$, respectively. The patients who underwent surgery had an ASA-PSC II or lower versus 6 of $16(37.5 \%)$ patients who received nonsurgical treatment $(p=0.008)$. Differences between the median times to recovery and median durations of hospitalization for the 2 patient groups were not significant. Infection of the surgical site occurred as a postoperative complication in only 1 patient, and there were no postoperative deaths or major complications. None of the patients who underwent surgery was readmitted. Twenty of the 36 (55.6\%) discharged patients who improved with nonsurgical treatment (including those receiving first drainage therapy only) were transferred to nursing homes or another hospital, and 5 of the 36 (13.9\%) discharged patients were readmitted because of recurrent empyema.

\section{DISCUSSION}

The increasing prevalence of acute empyema in elderly patients in poor overall clinical condition necessitates the consideration of nonsurgical treatment for these patients. In geriatric medicine, aspiration pneumonia is a common cause of respiratory infection. Indeed, 25 of 57 (43.9\%) of the patients analyzed in our study had dysphagia, which was a contraindication to surgical treatment. The infections of 43 of our patients improved after they underwent drainage procedures only (single or repeated). Furthermore, because we did not observe complications associated with CT-guided drainage, we think that the technique of inserting a pig-tail catheter into the pleural cavity is safe, although it is considered technically difficult. Our results indicate that the nonsurgical management of acute empyema by draining the pleural cavity and administering fibrinolytic therapy was a reasonable procedure alternative to surgery for elderly patients in poor overall clinical condition, because they are patients at high risk for surgery under general anesthesia.

Li et al. [10] reported on the utility of CT-guided drainage for empyema when the catheters were placed successfully and safely. Their study used catheters ranging from $8 \mathrm{Fr}$ to $14 \mathrm{Fr}$, which is similar to the size of the catheters used in our study.

A disadvantage of CT-guided drainage is that we cannot perform it at night or during holidays, because the CT-guided drainage technique is performed by a team of interventional radiologists to ensure accurate insertion of the drainage tube. Therefore, at our hospital the patients could not receive that treatment for a mean period of 26.2 hours. Based on our experience, with the exception of patients in septic shock, the treatment delay has not been a major problem. We only performed ultrasound-guided drainage for 2 emergency cases. Additionally, the accuracy and safety of CT-guided drainage overcame this disadvantage. A study performed in the United States found that patients undergoing nonsurgical treatment for acute empyema showed a higher readmission rate (7.3\%) than patients undergoing surgical treatment [11], which was confirmed by our study. Our readmission rate of $13.9 \%$ should be kept in mind when nonsurgical treatment is considered for a patient with acute empyema.

Clinicians who have never performed CT-guided drainage might be concerned that the small diameters of the catheters that are used would be inadequate for draining the pleural effusion. However, Rahman et al. [12] did not find significant relationships between the sizes of the chest tubes and clinical outcomes, with insignificant differences between the mortality rates or rates of patients needing thoracic surgery. The pain scores of patients treated with smaller tubes were lower, however [12]. 
The consensus guidelines of the American Association for Thoracic Surgery on acute empyema recommend routine flushing of the drains to prevent occlusion [1]; therefore, paying attention to the occlusion of smaller tubes is of increased importance. At our institution we often injected urokinase for 3 days through a pigtail catheter, which we assume also plays a role in flushing the drain, especially since no occluded drains were observed during the study period.

Although we frequently have administered fibrinolytic therapy, the routine use of this treatment remains controversial. We found that although the initial discharges from the drainage tubes were of relatively low volumes, the addition of intrapleural fibrinolytic therapy increased the efficacy of drainage. In their systematic review and meta-analysis of fibrinolytic therapy, Janda et al. [13] concluded that fibrinolytic therapy would be beneficial for patients with loculated pleural effusions, because it prevented the need for surgical intervention. Based on their results, we initially administered active fibrinolytic therapy to patients in poor overall clinical condition to reduce the risk of need for surgery as much as possible. We think that this approach was justified to some extent based on our results, especially for bedridden patients with acute empyema.

Streptokinase has been used mainly in the past for fibrinolytic therapy, while the combination of alteplase (t-PA) and DNase has been verified to be effective [14]. However, we cannot use either of these agents in Japan, and only use urokinase, which is popular here. The use of urokinase for fibrinolytic therapy is supported by the findings of Bedat et al. [15], who showed that urokinase was equally effective as t-PA/DNase and was safer with regard to the rate of hemothorax.

As a matter of course, we performed surgery for patients who were considered operable. The operative procedure was usually a VATS debridement, which included clearing all the loculations and removing all the pleural exudate. This procedure led to complete drainage of the pleural fluid. In this study, we performed surgery for patients with a PS of 2 or lower and an ASA-PSC of II or lower. With these criteria, complications did not occur during the perioperative period. Operable patients usually come to the hospital with minimal signs and symptoms, and their empyema is almost always in the exudative or fibropurulent phase. For this reason, we rarely needed to perform decortication of the lung, which is a difficult and invasive procedure [16]. We showed that none of the patients who underwent surgery was readmitted. Thus, we suggest that surgery is a radical cure for acute empyema. Thoracotomy was only performed for the rare patients with massive life-threatening ipsilateral pleural effusion or when differential lung ventilation was considered difficult to perform.

\section{Limitations}

Our study has limitations. First, the number of patients was relatively small, especially the patients who received additional treatment. Second, because of the retrospective design of the study and the fact that the patients undergoing surgical treatment were younger with better performance status and lower ASA-PSC compared with those parameters for the patients who underwent nonsurgical treatment, selection bias is possible. Third, our team consists of only two thoracic surgeons; thus, assessing the patients and scheduling the procedures were sometimes difficult. A large prospective study is needed to establish standard management guidelines for acute empyema.

\section{CONCLUSION}

For an aging population, the data suggest that the combination of repeated pleural cavity drainage procedures and fibrinolytic therapy is a reasonable nonsurgical strategy for the management of patients with acute empyema. We found that CT-guided drainage was a safe and effective procedure. Our study also found that surgery for acute empyema in patients with a PS of 2 or lower and an ASA-PSC of II or lower could be performed very safely.

\section{DISCLOSURES}

Acknowledgements

We gratefully acknowledge Masahiro Hirata (Center for Clinical and Translational Science, Shonan Kamakura General Hospital) for his advice. The authors also thank the staff at the Mirai Iryo Research Center Inc. for their advice.

\section{Funding}

Not applicable

\section{Competing interests}

The authors declare no conflict of interest.

\section{Ethics approval}

The study was approved by the Shonan Kamakura General Hospital Institutional Review Board (TGE01416-024).

\section{REFERENCES}

1. Finley C, Clifton J, Fitzgerald JM, Yee J. Empyema: an increasing concern in Canada. Can Respir J 2008;15:85-9. doi: 10.1155/2008/975312

2. Grijalva CG, Zhu Y, Nuorti JP, Griffin MR. The emergence of parapneumonic empyema in the USA. Thorax 2011;66:663-8. doi: 10.1136/ thx.2010.156406

3. Shen KR, Bribriesco A, Crabtree T, et al. The American Association for Thoracic Surgery consensus guidelines for the management of empyema. J Thorac Cardiovasc Surg 2017;153:e129-46. doi: 10.1016/j. jtcvs.2017.01.030

4. Scarci M, Abah U, Solli P, et al. EACTS expert consensus statement for surgical management of pleural empyema. Eur J Cardiothorac Surg 2015;48:642-53. doi: 10.1093/ejcts/ezv272

5. Reichert M, Hecker M, Witte B, et al. Stage-directed therapy of pleural empyema. Langenbecks Arch Surg 2017;402:15-26. doi: 10.1007/ s00423-016-1498-9

6. Sokouti M, Ghojazadeh M, Sokouti M, Sokouti B. Surgical and nonsurgical outcomes for treating a cohort of empyema thoracis patients: a monocenteric retrospective cohort study. Ann Med Surg 2017;24:19-24. doi: 10.1016/j.amsu.2017.10.002

7. Oken MM, Creech RH, Tormey DC, Horton J, Davis TE, McFadden ET. Toxicity and response criteria of the Eastern Cooperative Oncology Group. Am J Clin Oncol 1982;5:649-55. doi: 10.1097/00000421-198212000-00014

8. Driupps RD. New classification of physical status. Anesthesiology 1963;24:111.

9. Kanda Y. Investigation of the freely available easy-to-use software "EZR" for medical statistics. Bone Marrow Transplantation 2013;48:452-8. doi: 10.1038/bmt.2012.244

10. Li B, Liu C, Li Y, Yang HF, Du Y, Zhang C, et al. Computed tomography-guided catheter drainage with urokinase and ozone in management of empyema. World J Radiol 2017;9:212-16. doi: 10.4329/wjr.v9.i4.212

11. Semenkovich TR, Olsen MA, Puri V, Meyers BF, Kozower BD. Current state of empyema management. Ann Thorac Surg 2018;105:1589-96. doi: 10.1016/j.athoracsur.2018.02.027

12. Rahman NM, Maskell NA, Davies CW, et al. The relationship between chest tube size and clinical outcome in pleural infection. CHEST 2010;137:536-43. doi: 10.1378/chest.09-1044

13. Janda S, Swiston J. Intrapleural fibrinolytic therapy for treatment of adult parapneumonic effusions and empyemas: a systematic review and meta-analysis. Chest 2012;142:401-11. doi: 10.1378/chest.11-3071

14. Rahman NM, Maskell NA, West A, et al. Intrapleural use of tissue plasminogen activator and DNase in pleural infection. N Engl J Med 2011;365:518-26. doi: 10.1056/NEJMoa 1012740

15. Bédat B, Plojoux J, Noel J, et al. Comparison of intrapleural use of urokinase and tissue plasminogen activator/DNAse in pleural infection. ERJ Open Res 2019;5:00084-2019. doi: 10.1183/23120541.00084-2019

16. Tong BC, Hanna J, Toloza EM, et al. Outcomes of video-assisted thoracoscopic decortication. Ann Thorac Surg 2010;89:220-5. doi: 10.1016/j. athoracsur.2009.09.021 